\title{
¿Cómo motivar al adulto mayor durante su estimulación cognitiva con el uso de aplicaciones tecnológicas?
}

\author{
María Fernanda González Ross ${ }^{1}$, Christian O. Acosta Quiroz ${ }^{2}$, \\ Ramón René Palacio ${ }^{2}$, Joaquín Cortez ${ }^{1}$ \\ ${ }^{1}$ Instituto Tecnológico de Sonora, Unidad Nainari, \\ Sonora, México \\ ${ }^{2}$ Instituto Tecnológico de Sonora, Unidad Navojoa, \\ Sonora, México \\ mafher92@hotmail.com \\ \{joaquin.cortez, ramon.palacio, christian.acosta\}@itson.edu.mx
}

\begin{abstract}
Resumen. Este estudio se realizó con el fin de conocer cómo motivar al adulto mayor durante la estimulación cognitiva con el uso de aplicaciones tecnológicas. Para esto fue necesario entrevistar a ocho participantes tanto adultos mayores como personas que están a punto de ingresar a la tercera edad. Se les realizaron preguntas sobre lo que les gusta hacer, cómo les gustaría que los premiaran, entre otras. Dichas entrevistas tuvieron una duración de 30 minutos aproximadamente. Para la evaluación fue necesario analizar cada entrevista y obtener los puntos importantes para poder llegar al prototipo final que consta de un mensaje motivador para el adulto mayor y una conexión con sus familiares felicitándolos por el logro obtenido.
\end{abstract}

Palabras claves: Estimulación, adulto mayor, interacción humano-computadora.

\section{Introducción}

La finalidad de toda intervención en personas mayores es la de promover la estimulación, con el fin de extender la salud y favorecer una mayor valoración de las capacidades cognitivas para conservar el autoestima y la integración social del mayor en su propio entorno [1].

En todo el mundo, y México no es la excepción, se está viviendo un fenómeno interesante que es el envejecimiento de la población, cada vez la gente vive más y aparte cada vez hay más personas mayores. Entonces esto plantea retos importantes en todos los sentidos como en lo político, económico, educativo, en salud, etc. Esto ha aumentado el interés en problemáticas asociadas a la vejez como lo son:

- El deterioro cognitivo en funciones como la atención, memoria, concentración, solución de problemas, etc.

- Las enfermedades neurodegenerativas como las demencias tipo Alzheimer y las demencias vasculares. 
El Instituto Nacional de Estadística y Geografía [2], indica que en México se considera como adulto mayor a la persona mayor de 60 años. Este tipo de población son personas que han alcanzado madurez, los años vividos le han proporcionado la experiencia suficiente para solucionar de forma sabia cualquier situación, aunque físicamente agotado su organismo no responde igual, que en su juventud por todos los cambios que le han ocurrido. La imposibilidad de realizar actividades como las que ellos practicaban en sus años jóvenes pueden crearles complejos de inferioridad, baja autoestima, depresión, entre otros.

Estas personas llevan una vida productiva menor que la de los demás miembros de la familia, por tanto, el adulto mayor se convierte entonces en la persona inactiva dedicada a la casa y la búsqueda de los mandados después que ha dejado atrás una vida activa llena de relaciones amistosas, ha creado y formado una familia donde a él le corresponde un papel fundamental: ser la columna capaz de sustentar con sus consejos y experiencias.

Sin embargo en el ámbito de la tecnología el adulto mayor no es tomado en cuenta, porque se piensa que no son capaces de poder manejar aplicaciones, sistemas y videojuegos. Sin embargo desde hace mucho tiempo se ha señalado que los adultos mayores tienen la misma oportunidad de crecimiento, desarrollo, aprendizaje y el tener nueva experiencias como cualquier otra etapa de vida.

Por ello, es importante considerar los cambios que presentan en el envejecimiento, como son: las pérdidas sensoriales en la visión que se traducen en la reducción del campo visual, de la capacidad para distinguir pequeños detalles, del procesamiento de información visual y ajuste a la oscuridad, así como pérdidas auditivas que redundan en la reducción de la capacidad de escuchar ciertos sonidos o distinguir ciertas frecuencias, y de igual manera cambios en la movilidad que significan tiempos de respuestas más largos, reducción en habilidades motrices finas y una mayor fatiga. También, este tipo de población presenta cambios cognitivos que implican un decremento en la atención y pérdida de memoria remota y a corto plazo. Además, los adultos mayores experimentan dificultades al usar computadoras tales como requerir mayor tiempo para ejecutar ciertas actividades y para leer instrucciones e información textual, tienen más errores, olvidan el punto de la actividad que realizan, se confunden o no entienden el lenguaje técnico, se niegan a hacer algo que piensan que descompondrá la computadora y se incomodan o avergüenzan si algo sale mal [3].

Por ello, si se pretende desarrollar aplicaciones para el adulto mayor deben considerar aspectos tales como una guía constante a través de personajes virtuales, realimentación en la evaluación de las habilidades cognitivas, motivación después de cada ejercicio y graduar la dificultad de las actividades para mantener motivados a los adultos mayores [4].

En este estudio, particularmente nos enfocamos en uno de los aspectos anteriores que fue en la motivación para la utilización de alguna aplicación. La motivación es un aspecto importante para el adulto mayor porque si se encuentra motivado será concebida como un motor que impulsa hacia la vida, de igual manera pasa con la salud mental es la que mueve al cuerpo y además permite un razonamiento y conductas adecuadas en lo cotidiano. La motivación en el adulto mayor representa una gran energía impulsora que permite realizar cosas en la vida y disfrutarlas [4]. 
Para esto, es importante investigar qué factores resultan motivadores para el adulto mayor, lo cual pudo ser debido a que no se encontraron guías que evalúen la usabilidad y motivación de las aplicaciones para el adulto mayor.

En un estudio realizado por Theil, Schumacher, Adelsberger, Martin y Jäncke [5], los adultos mayores realizaron simultáneamente un entrenamiento cognitivo para mejorar su desempeño. Se realizaron veinte sesiones de 30 minutos cada una en un periodo de diez semanas, con una evaluación antes, a la mitad y después del entrenamiento. Los efectos del entrenamiento fueron evaluados con medidas de atención selectiva, asociación de pares, control ejecutivo, razonamiento, memoria, velocidad en el procesamiento de la información y ejecución de tareas motricescognitivas a manera de caminata y actividades de memoria. Participaron 63 adultos mayores con una edad media de 71.8 años (de 65 a 84 años), de los cuales 21 realizaron los dos entrenamientos simultáneos, 16 realizaron sólo el entrenamiento en memoria y 26 no llevaron a cabo entrenamiento alguno. Los resultados indican un progreso y mejora similar de tareas en control ejecutivo en ambos grupos con entrenamiento, esto en comparación con el grupo que no recibió entrenamiento alguno. Además, el entrenamiento simultáneo resultó en mejores logros en las tareas de asociación de pares en comparación al entrenamiento exclusivo en memoria. El entrenamiento simultáneo de capacidades cognitivas y físicas representan un concepto de entrenamiento promisorio para mejorar la ejecución de tareas cognitivas y motriz-cognitivas, ofreciendo mayor potencial en el funcionamiento cotidiano, el cual usualmente involucra múltiples capacidades y recursos, en vez de uno solo.

Como se puede notar, es posible apoyar al adulto mayor mediante entrenamiento, por lo que, la integración de mensajes motivadores en las aplicaciones serán importantes para el adulto mayor, pues ya han demostrado efectividad a la hora de querer estimular al adulto mayor, más si ese mensaje viene de parte de personas cercanas a ellos como sus familiares.

\section{Metodología}

Para este estudio se utilizó la metodología DesignThinking [6], la cual se basa en cinco fases: Empatizar, Definir, Idear, Prototipo y Prueba. El tipo de investigación fue cualitativa, pues los datos recolectados fueron por medio de unas entrevistas semiestructuradas que constaban de 25 preguntas sobre gustos, actividades, sentimientos del adulto mayor.

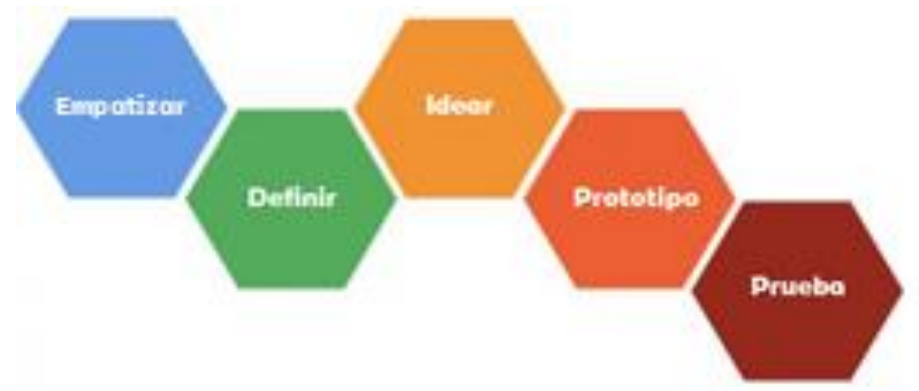

Fig. 1. Fases de la metodología DesignThinking. 
En la Fig. 1 se muestran las etapas de la metodología que se utilizó para realizar dicho estudio. Para esto se entrevistó a ocho adultos mayores de distintas partes, la duración de las entrevistas fue de aproximadamente de 30 minutos cada una, donde el adulto mayor podía expresar todo lo que pensaba con base en el tema que se le preguntaba como: qué conoce de tecnología, qué actividades realiza para entretenerse, de qué manera le gusta que se le premie entre otras. Para esto se le daba la confianza para así poder recibir la mayor información posible.

Dentro de los participantes se pudo destacar que hubo más participantes mujeres con un total de cinco. El promedio de edad del grupo fue de 63.5 años con una edad máxima de 85 años y una mínima de 60 años. Y cada participante presentaba diferentes deterioros de la vejez.

\subsection{Etapas del proceso de diseño}

Para realizar este estudio se tuvo que pasar por varias fases, la primera de ellas fue:

\section{Fase 1: Entrevistas}

En esta etapa se partió del problema para poder elaborar las 25 preguntas. Se construyó la entrevista basada en temas como: datos personales, vida social, pasatiempos, tecnologías, y videojuegos. Después de tener la entrevista se buscó a ocho personas mayores, que estuvieran dispuestas a contestar las preguntas se les comentaba que iba a durar aproximadamente 30 minutos eso causó un poco de molestias y varias personas se negaron a contestarlas porque comentaban que no tenían tiempo. Básicamente, este experimento fue realizado con entrevistas semi estructuradas las cuales nos ayudaron a conocer más sobre el adulto mayor y sus preferencias y así poder comprender sus gustos en cuanto los que se les motive o estimule cuando realizan determinada actividad.

Posteriormente, al tener las ocho entrevistas, se transcribieron todas las entrevistas para así poder analizar los datos obtenidos de cada participante. El siguiente paso fue a cada entrevista escrita colocar post-it a cada comentario que pensábamos que nos serviría o que considerábamos importante.

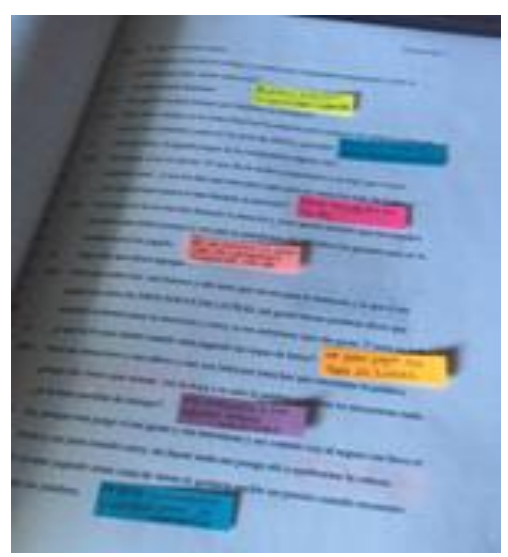

Fig. 2. Entrevista transcrita con post-it. 
En la Fig. 2 se muestra un ejemplo de la entrevista transcrita con los comentarios o datos relevantes en un post-it, esto fue para ir clasificando la información que finalmente nos ayudaría a encontrar como estimular o motivar al adulto mayor.

Después de eso, los post-it se pasaron a notas más grandes y se mezclaron con los demás post-it de las otras entrevistas para así poder categorizar cada comentario hecho por el adulto mayor (ver Fig. 3).

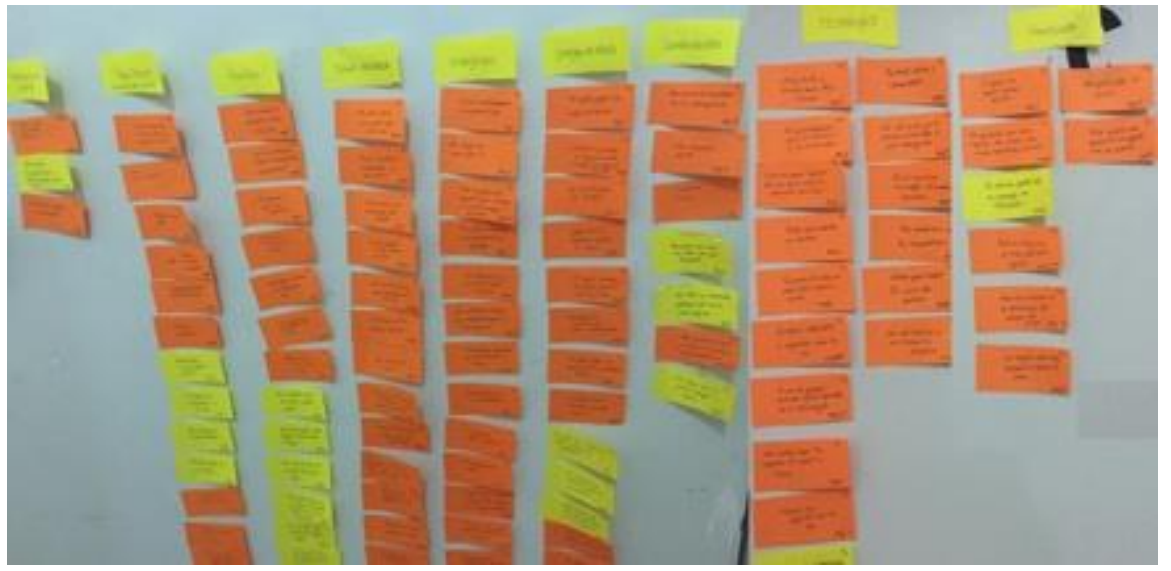

Fig. 3. Categorización de los comentarios de todas las entrevistas transcritas.
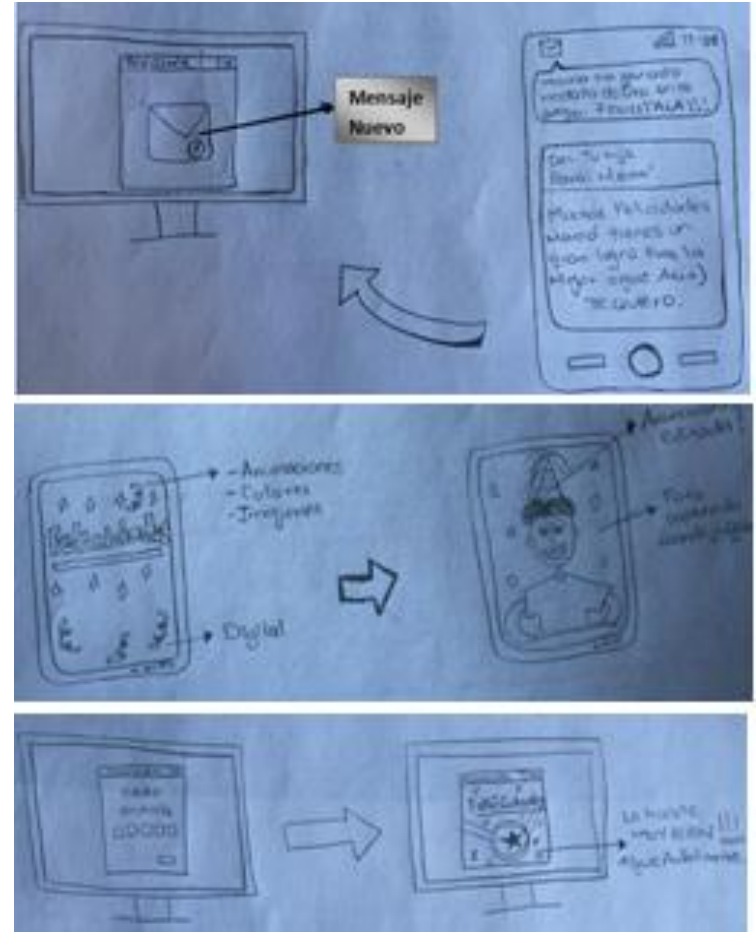

Fig. 4. Diseños basados en las respuestas dadas por el adulto mayor. 
Al analizar cada comentario de las entrevistas transcritas fue necesario pensar en categorías para ordenarlos. Salieron nueve categorías llamadas: Enfermedades, Pasatiempos, Familiares, Facebook, Videojuegos, Juegos de Mesa, Sentimientos, Tecnología y Premiación.

Posteriormente, todos esos comentarios categorizados se transcribieron en una tabla en Excel para tener un orden y así fuera más fácil analizar los comentarios.

Basándonos en estos resultados se construyeron varios diseños que prácticamente fueron obtenidos de las entrevistas realizadas al adulto mayor y así finalmente poder llegar a un prototipo final que cumpliera con las expectativas del adulto mayor.

\section{Fase 2: Generar diez diseños}

En esta fase, después de haber realizado las entrevistas y analizado los comentarios hechos por el adulto mayor, se generaron diez diseños basados en los resultados que se obtuvieron de las entrevistas. Los diseños fueron hechos a mano con papel y lápiz, algo sencillo pero entendible ya que esto nos sirvió para el siguiente paso. A continuación se muestran algunos en la Fig. 4.

En dicha Figura se muestran los diseños que al adulto mayor le gustaría percibir y que creen ellos que eso pudiera motivarlos para seguir haciendo uso de alguna aplicación.

\section{Fase 3: Reducir a tres diseños utilizando las técnicas}

La siguiente fase fue reducir los diseños de diez a tres. Para esto se siguió utilizando el diseño con papel y lápiz, sólo que los diseños fueron de una forma más detallada. Y se utilizaron varias técnicas como: Sketching, storyboarding.

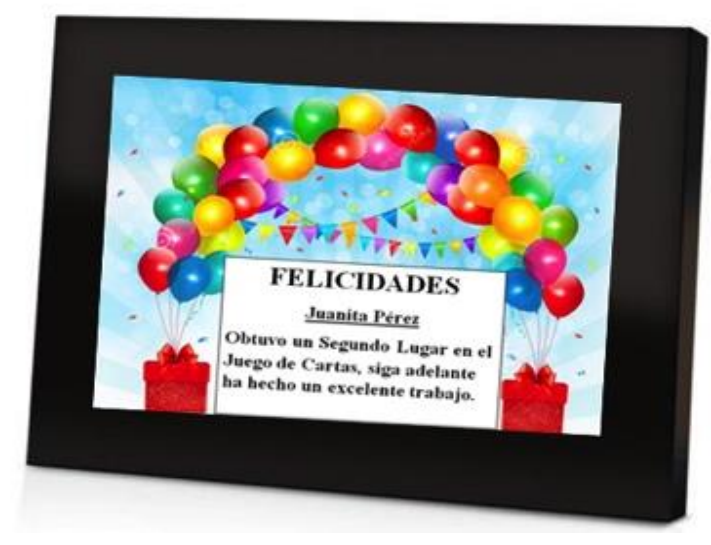

Fig. 5. Porta retrato con el logro que obtuvo el adulto mayor.

Este diseño (Fig. 5) fue poner su logro en un porta retrato ya sea digital o normal y así el adulto mayor podrá tener su logro junto con las fotos importantes de sus familiares. Pero las desventajas que vimos en él fue que al tener varios reconocimientos iba a ocupar mucho espacio y también probablemente sus familiares ya no le iban a tomar tanta importancia como al primero. Y eso podía causar desanimo en el adulto mayor y no resultaría que siguieran utilizando tal aplicación que cuente con este tipo de reconocimiento. 


\subsection{Descripción del prototipo}

Finalmente se llegó a un prototipo final, el cual se basó en las respuestas que proporcionaron los adultos mayores al momento de entrevistarlos.

El objetivo del estudio fue cómo le gustaría al adulto mayor que se les motivara o qué les gustaría obtener para motivarlos, la mayoría respondió que un mensaje que los incentive a seguir adelante los hará sentir muy bien. Después se analizó qué más se podría hacer para que ellos se sintieran cómodos y en confianza. Al seguir analizando las entrevistas se notó que el adulto mayor lo más importante en su vida son sus familiares y estar en contacto con ellos, pero por varias circunstancias muchas veces no es posible. Fue por eso que se pensó el diseño del prototipo y se diseñó de esta manera.

En varias entrevistas realizadas los adultos mayores hicieron comentarios como:

"Me gustaría que al tener un logro pues me felicitaran por medio de un mensaje motivador donde me digan que lo hice bien y que siga echándole ganas para así yo saber que aun puedo superarme y aprender o hacer cosas nuevas"

[Participante 3].

Esto ayudó a diseñar el siguiente prototipo que pueda ser usado en cualquier tecnología como: aplicaciones de entretenimiento, recordatorios de medicamentos, entre otras. Básicamente consistiría en que al final se les premiará con un mensaje dependiendo de la puntuación obtenida, pero siempre animándolos para que siga adelante.

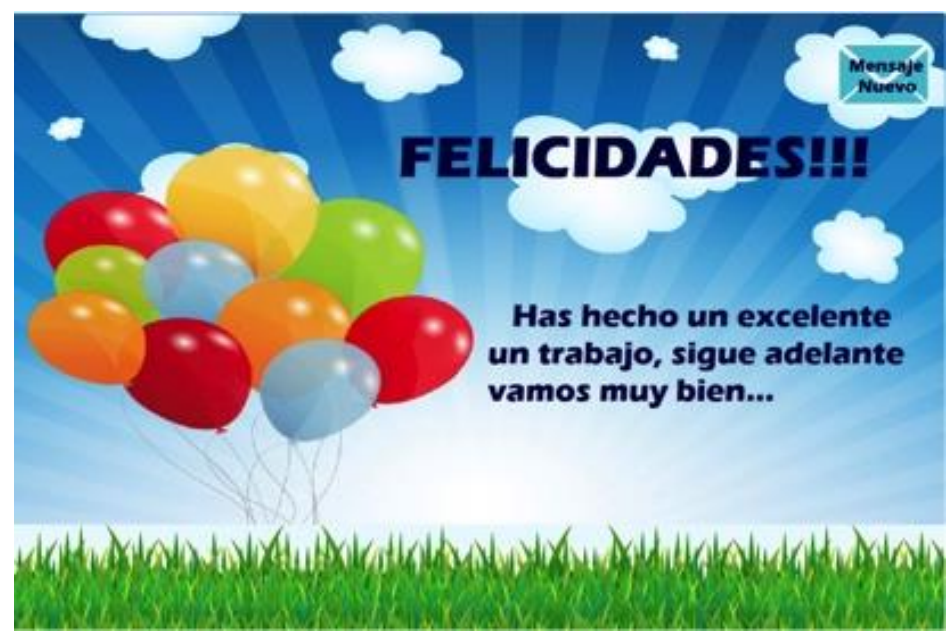

Fig. 6. Prototipo final - Pantalla principal.

En la Fig. 6 se muestra como les aparecería el mensaje en la pantalla con colores del agrado del adulto mayor y considerando una letra de tamaño 16 y fuente legible.

Ahora tratando de abordar cómo se podría hacer la relación del adulto mayor con su familiar más cercano, ya que en dichas entrevistas ellos comentaban que lo más importante eran sus familiares y la relación o el tiempo que podían estar en contacto con ellos. Y fue ahí donde elaboró el diseño mostrado en la Fig. 7. 


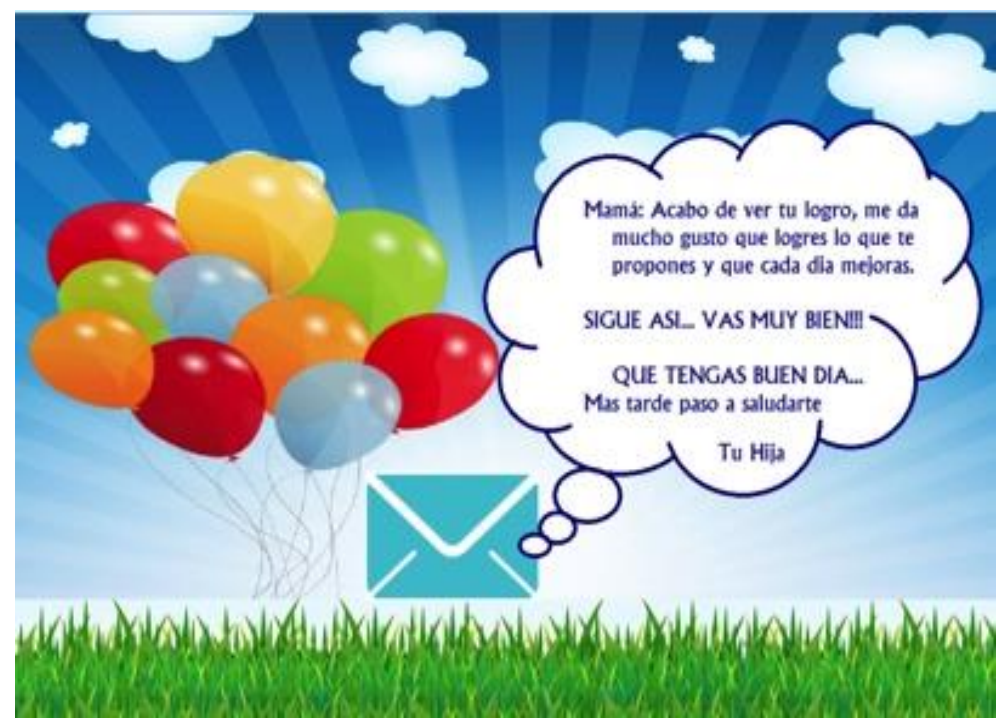

Fig. 7. Prototipo final-Segunda pantalla.

En este diseño lo que se pretende hacer es al terminar de utilizar alguna aplicación para el adulto mayor automáticamente el sistema mande un mensaje al celular del familiar poniéndolo al tanto de que su mamá, abuela, etc., ha tenido un logro en la aplicación. Posteriormente, el familiar deberá contestar el mensaje en el celular y enviarlo, este mensaje será recibido por el sistema y lo mostrará en pantalla.

El adulto mayor podrá visualizar el mensaje de su familiar, con lo que se esperaría se sienta motivado, ya que su familia estaría en contacto y al pendiente de él o ella. Esto podría ayudar a que el adulto mayor quiera seguir utilizando la aplicación y se abordarían varios factores importantes para el adulto como lo es la atención, memoria, razonamiento y planificación.

Este tipo de mensajes para las aplicaciones es posible desarrollar mediante la plataforma Unity 5 para Microsoft Windows y el Framework de Visual Studio .NET 2012. Esta aplicación deberá utilizar Servicios Web con el cual se enviarían al servidor las peticiones, ya que con dichos servicios será posible enviar información referente al adulto mayor y sus logros en la aplicación que utiliza a los familiares vía correo electrónico o incluso vía mensaje de texto (SMS). Con esto los familiares podrían realimentar al adulto mayor contestándole por la vía que haya recibido la información.

Para poder diseñar y llegar al prototipo final fue necesario analizar una y otra vez las entrevistas para tomar los puntos importantes que considera en su vida el adulto mayor y adecuarlos al diseño.

\section{Conclusiones}

En conclusión, este trabajo sirvió para obtener el diseño para estimular al adulto mayor a que utilice aplicaciones que pueden desarrollarse para ellos y realmente se sientan cómodos y quieran seguirlas usando por mayor tiempo. Sin embargo, trabajar con adultos mayores es un gran reto ya que en esta etapa de la vida, algunos se 
encuentran decaídos, desatendidos por sus familiares y eso implica un gran desanimo en el adulto mayor.

Este trabajo informa los resultados obtenidos del estudio, donde se obtuvieron nueve categorías que salieron de las entrevistas realizadas al adulto mayor. Para ello fue necesario analizar cada una de ellas para así poder categorizar de la siguiente manera: enfermedades, pasatiempos, familiares, Facebook, videojuegos, juegos de mesa, sentimientos, tecnologías, premiación. Las categorías más importantes fueron: premiación, sentimientos, tecnología, familiares, pasatiempo. Las categorizas que no tuvieron mucho significado para el adulto mayor fueron la de Facebook, videojuegos, juego de mesa, y enfermedades.

La categorización de las respuestas del adulto mayor sirvió para poder tomar en cuenta todas las opiniones que el adulto mayor piensa o siente y así diseñar algo que realmente sea motivador para ellos. Se analizaron todos los diseños resultantes, haciendo comparaciones y con las mismas opiniones del adulto mayor y fue así como se llegó al diseño final, el cual resultó un mensaje motivador que los haga seguir adelante y tener el contacto con sus familiares y que ellos estén al pendiente de lo que están realizando.

Este diseño puede ser utilizado para cualquier aplicación enfocada al adulto mayor ya que sería la parte final de las aplicaciones.

Esperemos que prototipo final que se presentó anteriormente sea de su agrado y así esto ayude a complementar aplicaciones en cuanto a cómo estimular al adulto mayor mediante el uso de mensajes de motivación. Como trabajo futuro se pretende desarrollar un prototipo de este tipo para que puedan integrarse a aplicaciones para el adulto mayor y así evaluar la experiencia de uso y usabilidad.

\section{Referencias}

1. Yuni, J.A., Urbano, C.A.: Envejecimiento y género: perspectivas teóricas y aproximaciones al envejecimiento femenino. Revista argentina de sociología, Vol. 6, pp. 151-169 (2008)

2. Instituto Nacional de Estadistica, G.e.I.: Estadisticas sobre disponibilidad y uso de tecnologia de informacion y comunicacion en los hogares. pp. 8-17, México (2005)

3. Rute-Pérez, S., Santiago-Ramajo, S., Hurtado, M.V., Rodríguez-Fórtiz, M.J., Caracuel, A.: Challenges in software applications for the cognitive evaluation and stimulation of the elderly. Journal of neuroengineering and rehabilitation 11, 88 (2014)

4. Rebok, G.W., Langbaum, J.B., Jones, R.N., Gross, A.L., Parisi, J.M., Spira, A.P., Kueider, A.M., Petras, H., Brandt, J.: Memory Training in the ACTIVE Study How Much is Needed and Who Benefits? Journal of aging and health 25, 21S-42S (2013)

5. Theill, N., Schumacher, V., Adelsberger, R., Martin, M., Jäncke, L.: Effects of simultaneously performed cognitive and physical training in older adults. BMC neuroscience $14,103(2013)$

6. Steinbeck, R.: El «design thinking» como estrategia de creatividad en la distancia. Comunicar 19, pp. 27-35 (2011) 